Journal of Systems Science and Information

Jun., 2017, Vol. 5, No. 3, pp. 229-249

DOI: $10.21078 /$ JSSI-2017-229-21

\title{
Portfolio Selection with Random Liability and Affine Interest Rate in the Mean-Variance Framework
}

\author{
Hao CHANG \\ College of Management and Economics, Tianjin University, Tianjin 300072, China; School of \\ Science, Tianjin Polytechnic University, Tianjin 300387, China \\ E-mail: ch8683897@126.com \\ Chunfeng WANG \\ College of Management and Economics, Tianjin University, Tianjin 300072, China \\ E-mail: cfwang@tju.edu.cn \\ Zhenming FANG \\ College of Management and Economics, Tianjin University, Tianjin 300072, China \\ E-mail: zmfang@tju.edu.cn
}

\begin{abstract}
This paper studies a dynamic mean-variance portfolio selection problem with random liability in the affine interest rate environment, where the financial market consists of three assets: one risk-free asset, one risky asset and one zero-coupon bond. Assume that short rate is driven by affine interest rate model and liability process is described by the drifted Brownian motion, in addition, stock price dynamics is affected by interest rate dynamics. The investors expect to look for an optimal strategy to minimize the variance of the terminal surplus for a given expected terminal surplus. The efficient strategy and the efficient frontier are explicitly obtained by applying dynamic programming principle and Lagrange duality theorem. A numerical example is given to illustrate our results and some economic implications are analyzed.
\end{abstract}

Keywords affine interest rate; random liability; mean-variance criterion; the efficient strategy; the efficient frontier; Lagrange duality theorem

\section{Introduction}

Mean-variance criterion and utility criterion are two fundamental methods dealing with the portfolio selection problems in the modern financial theory. It is all well-known that the pioneering work on the mean-variance portfolio selection problem was done by Markowitz ${ }^{[1]}$. But the optimal asset allocation problem that Markowitz considered was a simple and singleperiod discrete-time one and was studied in a static mean-variance framework. Therefore, multiperiod and continuous-time dynamic portfolio choice problems have attracted great attentions of

Received May 23, 2016, accepted December 19, 2016

Supported by National Natural Science Foundation of China (71671122), China Postdoctoral Science Foundation Funded Project (2014M560185, 2016T90203), Humanities and Social Science Research Fund of Ministry of Education of China (11YJC790006, 16YJA790004) and Tianjin Natural Science Foundation of China (15JCQNJC04000) 
many authors since Markowitz. Bajeux-Besnainou and Portait ${ }^{[2]}$ used a martingale approach to investigate a continuous-time dynamic mean-variance problem. $\mathrm{Li}$ and $\mathrm{Ng}^{[3]}$ developed a linear quadratic (LQ) control approach and for the first time successfully solved the meanvariance portfolio selection problem in a multi-period environment. Zhou and $\mathrm{Li}^{[4]}$ applied the LQ approach to a continuous-time mean-variance portfolio selection problem and obtained the closed-form solutions to the efficient strategy and the efficient frontier. Fu, et al. ${ }^{[5]}$ used dynamic programming (DP) principle and Lagrange duality theorem to solve a continuoustime mean-variance problem with borrowing constraints and successfully derived the closedform solutions to the optimal policy and the efficient frontier. Lim and Zhou ${ }^{[6]}$ and Ferland and Watier ${ }^{[7]}$ applied the backward stochastic differential equation (BSDE) theory to the meanvariance portfolio selection problems with random parameters and with extended CIR interest rates respectively. Those research results laid down solid foundations for further investigating the mean-variance portfolio selection problems. In summary, there are four main techniques in dealing with mean-variance portfolio selection problems: (i) The martingale approach; (ii) The LQ approach, (iii) DP principle and Lagrange duality theorem, (iv) BSDE theory.

In recent years, the asset and liability management (ALM) problems have been paid more and more attentions to. As a matter of fact, liability factor is often confronted with in the activity of investment. Some investors realized that it was very clear that the optimal strategies with liability environments are more practical. Therefore, it is very necessary for us to introduce liability factors into dynamic portfolio selection problems and investigate the optimal investment strategy. We can summarize research results on the ALM problems as follows. (i) Multi-period ALM problems. For example, Yi, et al. ${ }^{[8]}$ studied an ALM problem with uncertain investment horizon, Chen and Yang ${ }^{[9]}$ investigated an ALM problem with regime switching, Yao, et al. ${ }^{[10]}$ considered an ALM problem with uncontrolled cash flow and uncertain horizon, Leippold, et al. ${ }^{[11]}$ focused on an ALM problem with endogenous liability, and so on. (ii) Continuous-time ALM problems. For instance, Xie, et al. ${ }^{[12]}$ studied an ALM problem by assuming liability process to be driven by the drifted Brownian motion, Zeng and $\mathrm{Li}^{[13]}$ assumed liability process to be governed by Brownian motion with jump and investigated the optimal policy and the efficient frontier, Chen, et al. ${ }^{[14]}$ devoted themselves to the ALM problem with regime switching, Yao, et al. ${ }^{[15]}$ discussed the ALM problem with endogenous liabilities. These research results greatly enriched and expanded the ALM theories and laid down solid foundations for solving more sophisticated and realistic ALM problems. The concern was that the research results mentioned above were achieved under the assumption of constant interest rate.

It is all well-known that interest rate isn't always fixed. Ever since the oil crisis in the last century, the interest rate has appeared more volatile in the many western countries. Therefore, it is very reasonable to incorporate stochastic interest rate model into the dynamic portfolio selection problems. In addition, it is very obvious that the optimal investment strategy under stochastic interest rate model will be more practical. Previous works on dynamic portfolio selection problems with stochastic interest rates included Korn and Kraft ${ }^{[16]}$, Deelstra, et al. ${ }^{[17]}$, $\mathrm{Gao}^{[18]}$, Chang, et al. ${ }^{[19]}, \mathrm{Li}$ and $\mathrm{Wu}^{[20]}$, Liu ${ }^{[21]}$, Chang and Rong ${ }^{[22]}$, Guan and Liang ${ }^{[23,24]}$. Those models were all studied in the utility maximization framework. As far as we know, it is very difficult to derive the explicit expressions of the optimal policies and the efficient frontier 
in investigating dynamic mean-variance portfolio selection problems with stochastic interest rates. In the existing literatures, only few authors, for example, Lim and Zhou ${ }^{[6]}$ and Ferland and Watier ${ }^{[7]}$, successfully solved those problems, but the approach they used is just the BSDE theory. But if we introduce liability process into dynamic mean-variance portfolio selection problems with stochastic interest rates, it will give rise to many difficulties in constructing the BSDE such that we have no ways to obtain the closed-form solutions to the efficient strategy and the efficient frontier.

As a matter of fact, stochastic optimal control theory is also an effective approach in dealing with dynamic mean-variance portfolio selection problems with stochastic interest rates. In this paper, we introduce liability process into a dynamic mean-variance portfolio selection problem and assume interest rate to be driven by affine interest rate model, which includes the Vasicek model and the CIR model as special cases. Liability process is supposed to be governed by the drifted Brownian motion. The surplus is expected to be allocated in the three financial assets, which consists of one risk-free asset, one risky asset and one zero-coupon bond. The objective of the investors is to minimize the variance of the terminal surplus for a given the expectation of terminal surplus. In this paper, dynamic programming principle is used to derive the optimal investment strategies. After successfully solving the equation (22), we obtain the closed-form solutions to the optimal policies. By applying Lagrange duality theorem, we obtain the explicit expression of the efficient frontier. As a result, a numerical example is provided to illustrate these results. There have three main contributions in this paper: (i) We introduce liability factor into a dynamic portfolio selection problem with affine interest rate; (ii) We successfully solve the mean-variance model with stochastic interest rate; (iii) We use dynamic programming principle and Lagrange duality theorem altogether to successfully obtain the closed-form solutions to the efficient strategies and the efficient frontier.

The paper proceeds as follows. Section 2 describes the problem framework. In Section 3, we use Lagrange multiplier technique and dynamic programming principle to obtain the closedform solution to the optimal policies. In Section 4, we obtain the explicit expression of the efficient frontier by applying Lagrange duality theorem. Section 5 gives a numerical example to demonstrate our results and Section 6 concludes the paper.

\section{The Model}

In this section, we present the problem formulation, which is composed of four parts: The financial market, the liability process, the wealth process and the optimization criterion. Assume that the financial market consists of three financial assets: One risk-free asset, one risky asset and one zero-coupon bond. In addition, an investor can buy or sell continuously without incurring any restriction as short-selling constraint or borrowing constraint or any transaction costs.

Assume that $\left(W_{r}(t), W_{S}(t)\right)$ is a two-dimension standard and adapted independent Brownian motion defined on complete probability space $\left(\Omega, \mathscr{F}, \mathbb{P},\left\{\mathscr{F}_{t}\right\}_{t \in[0, T]}\right)$, where $\mathbb{P}$ is the real world probability and the filtration $\left\{\mathscr{F}_{t}\right\}_{t \in[0, T]}$ is the information structure generated by Brownian motion $\left(W_{r}(t), W_{S}(t)\right)$. $[0, T]$ represents the finite and fixed investment horizon.

The first asset is a risk-free asset (i.e., cash or bank account), whose price at time $t$ is 
denoted by $S_{0}(t), t \in[0, T]$, then $S_{0}(t)$ evolves according to

$$
\mathrm{d} S_{0}(t)=r(t) S_{0}(t) \mathrm{d} t, \quad S_{0}(0)=1,
$$

where short rate dynamics $r(t)$ is described by the following stochastic differential equation (SDE):

$$
\mathrm{d} r(t)=(a-b r(t)) \mathrm{d} t-\sqrt{k_{1} r(t)+k_{2}} \mathrm{~d} W_{r}(t), \quad r(0)=r_{0}>0,
$$

where $a, b, k_{1}$ and $k_{2}$ are positive constants.

Remark 1 SDE (2) have been investigated by Deelstra, et al..17], Gao ${ }^{[18]}$ and Chang, et al. ${ }^{[19]}$. Their papers show that term structure of interest rate is affine under SDE (2). In addition, the Vasicek ${ }^{[25]}$ (resp. CIR model ${ }^{[26]}$ ) dynamics is one of special case of these dynamics, when $k_{1}$ (resp. $k_{2}$ ) is equal to zero.

The second asset is the stock. Its price, denoted by $S_{1}(t), t \in[0, T]$, is given by (referring to Deelstra, et al. ${ }^{[17]}$, Gao ${ }^{[18]}$ and Chang, et al. ${ }^{[19]}$ ):

$$
\begin{aligned}
\frac{\mathrm{d} S_{1}(t)}{S_{1}(t)}= & r(t) \mathrm{d} t+\sigma_{1}\left(\mathrm{~d} W_{S}(t)+\lambda_{1} \mathrm{~d} t\right) \\
& +\sigma_{2} \sqrt{k_{1} r(t)+k_{2}}\left(\mathrm{~d} W_{r}(t)+\lambda_{2} \sqrt{k_{1} r(t)+k_{2}} \mathrm{~d} t\right),
\end{aligned}
$$

with initial condition $S_{1}(0)=1$ and $\lambda_{1}, \lambda_{2}$ (resp. $\sigma_{1}, \sigma_{2}$ ) being constants (resp. positive constants), see Deelstra, et al. ${ }^{[17]}, \mathrm{Gao}^{[18]}$ and Chang, et al..19].

The third asset is a zero-coupon bond with maturity $T$, whose price is denoted by $B(t, T)$. The dynamics of $B(t, T)$ is described by the following SDE (referring to [17-19]):

$$
\frac{\mathrm{d} B(t, T)}{B(t, T)}=r(t) \mathrm{d} t+\sigma_{B}(t, r(t))\left(\mathrm{d} W_{r}(t)+\lambda_{2} \sqrt{k_{1} r(t)+k_{2}} \mathrm{~d} t\right), \quad B(T, T)=1,
$$

where

$$
\begin{aligned}
& \sigma_{B}(t, r(t))=h(t) \sqrt{k_{1} r(t)+k_{2}}, \\
& h(t)=\frac{2\left(\mathrm{e}^{m(T-t)}-1\right)}{m-\left(b-k_{1} \lambda_{2}\right)+\mathrm{e}^{m(T-t)}\left(m+b-k_{1} \lambda_{2}\right)}, \\
& m=\sqrt{\left(b-k_{1} \lambda_{2}\right)^{2}+2 k_{1}} .
\end{aligned}
$$

Suppose that an investor has the exogenous liability $L(t)$ at time $t, t \in[0, T]$, then $L(t)$ can be governed by the drifted Brownian motion:

$$
\mathrm{d} L(t)=u \mathrm{~d} t+v \mathrm{~d} W_{S}(t), \quad L(0)=l_{0}>0,
$$

where $u$ and $v$ are positive constants.

Assume that an investor is equipped with the initial wealth $w_{0}>0$ and has initial liability $l_{0}>0$ at $t=0$, then the initial surplus of the investor is given by $x_{0}=w_{0}-l_{0}>0$. The surplus process at time $t$ is denoted by $X(t)$, and the capital amount invested the stock and the zero-coupon bond is denoted by $\pi_{1}(t)$ and $\pi_{2}(t)$ respectively. Under the investment strategy $\pi(t)=\left(\pi_{1}(t), \pi_{2}(t)\right)$, the surplus process $X(t)$ satisfies the following dynamics:

$$
\begin{aligned}
\mathrm{d} X(t)= & \left(X(t) r(t)+\pi_{1}(t) \sigma_{1} \lambda_{1}+\pi_{1}(t) \sigma_{2} \lambda_{2}\left(k_{1} r(t)+k_{2}\right)+\pi_{2}(t) \sigma_{B} \lambda_{2} \sqrt{k_{1} r(t)+k_{2}}-u\right) \mathrm{d} t \\
& +\left(\pi_{1}(t) \sigma_{1}-v\right) \mathrm{d} W_{S}(t)+\left(\pi_{1}(t) \sigma_{2} \sqrt{k_{1} r(t)+k_{2}}+\pi_{2}(t) \sigma_{B}\right) \mathrm{d} W_{r}(t),
\end{aligned}
$$


with the initial condition $X(0)=x_{0}>0$.

Definition 1 (Admissible strategy) An investment strategy $\left(\pi_{1}(t), \pi_{2}(t)\right)$ is said to be admissible if the following conditions are satisfied:

(i) $\left(\pi_{1}(t), \pi_{2}(t)\right)$ is $\mathscr{F}_{t}$-progressively measurable, and

$$
\int_{0}^{T} \pi_{1}^{2}(t) \mathrm{d} t<\infty, \quad \int_{0}^{T} \pi_{2}^{2}(t) \mathrm{d} t<\infty
$$

(ii)

$$
E\left(\int_{0}^{T}\left(\left(\pi_{1}(t) \sigma_{1}-v\right)^{2}+\left(\pi_{1}(t) \sigma_{2} \sqrt{k_{1} r(t)+k_{2}}+\pi_{2}(t) \sigma_{B}\right)^{2}\right) \mathrm{d} t\right)<\infty ;
$$

(iii) The SDE (6) has an unique solution under the investment strategy $\pi(t)=\left(\pi_{1}(t), \pi_{2}(t)\right)$.

We denote the set of all admissible strategies $\pi(t)=\left(\pi_{1}(t), \pi_{2}(t)\right)$ by $\Gamma$, The investors' objective is to find an optimal portfolio $\left(\pi_{1}(t), \pi_{2}(t)\right)$ such that the expected terminal surplus satisfies $E(X(T))=C$, for some constant $C \in \mathbb{R}$, while the risk measured by the variance of the terminal surplus

$$
\operatorname{Var} X(T)=E(X(T)-E(X(T)))^{2}=E(X(T)-C)^{2}
$$

is minimized. The problem of finding such a portfolio $\left(\pi_{1}(t), \pi_{2}(t)\right)$ is referred to as the meanvariance portfolio choice problem.

Therefore the mean-variance model can be formulated as a linearly constrained stochastic optimization problem:

$$
\begin{aligned}
\min _{\pi(t) \in \Gamma} & \operatorname{Var} X(T)=E(X(T)-C)^{2} \\
\text { s.t. } & E(X(T))=C .
\end{aligned}
$$

Finally, an optimal investment strategy of the above problem is called an efficient portfolio corresponding to some constant $C \in \mathbb{R}$, and the corresponding $(C, \operatorname{Var} X(T))$ is called an efficient point, whereas the set of all the efficient points is called the efficient frontier.

\section{The Optimal Portfolios}

To find the optimal investment strategy for the problem (8) corresponding to the constraint $E(X(T))=C$, we introduce a Lagrange multiplier $2 \lambda \in \mathbb{R}$ and arrive at the new objective function:

$$
\hat{L}\left(\pi_{1}(t), \pi_{2}(t), \lambda\right)=E\left((X(T)-C)^{2}+2 \lambda(X(T)-C)\right)=E(X(T)-(C-\lambda))^{2}-\lambda^{2} .
$$

Letting $\eta=C-\lambda$, we obtain the following stochastic control problem:

$$
\min \quad \bar{L}\left(\pi_{1}(t), \pi_{2}(t), \eta\right)=E(X(T)-\eta)^{2}-(C-\eta)^{2} .
$$

The link between the problems (9) and (11) is provided by Lagrange duality theorem (see Fu, et al. $\left.{ }^{[5]}\right)$, namely, we have

$$
\min \operatorname{Var} X(T)=\max _{\lambda \in R} \min _{\pi(t) \in \Gamma} \hat{L}\left(\pi_{1}(t), \pi_{2}(t), \lambda\right)=\max _{\eta \in R} \min _{\pi(t) \in \Gamma} \bar{L}\left(\pi_{1}(t), \pi_{2}(t), \eta\right) .
$$


For fixed constants $\eta$ and $C$, the problem (11) is clearly equivalent to

$$
\min _{\pi(t) \in \Gamma} E(X(T)-\eta)^{2}
$$

We define the value function $H(t, r, x)$ as

$$
H(t, r, x)=\min _{\pi(t) \in \Gamma} E\left((X(T)-\eta)^{2} \mid r(t)=r, X(t)=x\right)
$$

with the boundary condition $H(T, r, x)=(x-\eta)^{2}$.

For any function $H(t, r, x) \in \mathbb{C}^{1,2,2}([0, T] \times \mathbb{R} \times \mathbb{R})$, we define a variational operator:

$$
\begin{aligned}
\mathcal{A}^{\pi(t)} H(t, r, x)= & H_{t}+\left(r x+\pi_{1}(t) \sigma_{1} \lambda_{1}+\pi_{1}(t) \sigma_{2} \lambda_{2} \sigma_{r}^{2}+\pi_{2}(t) \sigma_{B} \lambda_{2} \sigma_{r}-u\right) H_{x} \\
& +\frac{1}{2}\left(\left(\pi_{1}(t) \sigma_{1}-v\right)^{2}+\left(\pi_{1}(t) \sigma_{2} \sigma_{r}+\pi_{2}(t) \sigma_{B}\right)^{2}\right) H_{x x}+\frac{1}{2} \sigma_{r}^{2} H_{r r} \\
& +(a-b r) H_{r}-\left(\pi_{1}(t) \sigma_{2} \sigma_{r}^{2}+\pi_{2}(t) \sigma_{B} \sigma_{r}\right) H_{r x}
\end{aligned}
$$

where $\sigma_{r}=\sqrt{k_{1} r(t)+k_{2}}$, and $H_{t}, H_{x}, H_{x x}, H_{r}, H_{r r}, H_{r x}$ represent first-order and second-order partial derivatives with respect to the variables $t, r, x$, respectively.

According to the conclusion obtained by Fleming and Soner ${ }^{[27]}$, we can derive the following HJB equation for the problem (14):

$$
\min _{\pi(t) \in \Gamma}\left\{\mathcal{A}^{\pi(t)} H(t, r, x)\right\}=0
$$

with terminal condition $H(T, r, x)=(x-\eta)^{2}$.

Differentiating (16) with respect to $\pi_{1}(t)$ and $\pi_{2}(t)$ respectively, we derive

$$
\begin{aligned}
& \pi_{1}^{*}(t)=-\frac{\lambda_{1}}{\sigma_{1}} \cdot \frac{H_{x}}{H_{x x}}+\frac{v}{\sigma_{1}}, \\
& \pi_{2}^{*}(t)=\frac{\sigma_{r}}{\sigma_{B}} \cdot \frac{\sigma_{2} \lambda_{1}-\sigma_{1} \lambda_{2}}{\sigma_{1}} \cdot \frac{H_{x}}{H_{x x}}+\frac{\sigma_{r}}{\sigma_{B}} \cdot \frac{H_{r x}}{H_{x x}}-\frac{\sigma_{2}}{\sigma_{1}} \cdot \frac{\sigma_{r}}{\sigma_{B}} v .
\end{aligned}
$$

Substituting (17) and (18) back into (16), we have

$$
\begin{aligned}
& H_{t}+\left(r x+\lambda_{1} v-u\right) H_{x}+(a-b r) H_{r}+\frac{1}{2} \sigma_{r}^{2} H_{r r} \\
& -\frac{1}{2}\left(\lambda_{1}^{2}+\lambda_{2}^{2} \sigma_{r}^{2}\right) \frac{H_{x}^{2}}{H_{x x}}+\lambda_{2} \sigma_{r}^{2} \frac{H_{x} H_{r x}}{H_{x x}}-\frac{1}{2} \sigma_{r}^{2} \frac{H_{r x}^{2}}{H_{x x}}=0
\end{aligned}
$$

with terminal condition: $H(T, r, x)=(x-\eta)^{2}$.

We try to conjecture $H(t, r, x)$ with the following structure

$$
H(t, r, x)=f(t, r)(x-g(t, r))^{2}, \quad f(T, r)=1, \quad g(T, r)=\eta
$$

The partial derivatives with respect to $H(t, r, x)$ are following:

$$
\begin{aligned}
& H_{t}=f_{t}(x-g)^{2}-2 f(x-g) g_{t}, \quad H_{x}=2 f(x-g), \quad H_{x x}=2 f, \\
& H_{r}=f_{r}(x-g)^{2}-2 f(x-g) g_{r}, \quad H_{r x}=2 f_{r}(x-g)-2 f g_{r},
\end{aligned}
$$




$$
H_{r r}=f_{r r}(x-g)^{2}-4(x-g) f_{r} g_{r}+2 f g_{r}^{2}-2 f(x-g) g_{r r} .
$$

Putting the above partial derivatives into (19) and considering

$$
\left(r x+\lambda_{1} v-u\right) H_{x}=2 r f(x-g)^{2}+2\left(r g+\lambda_{1} v-u\right) f(x-g) .
$$

After some simplification, we obtain

$$
\begin{aligned}
& (x-g)^{2}\left(f_{t}+\left(2 r-\lambda_{1}^{2}-\lambda_{2}^{2} \sigma_{r}^{2}\right) f+\left(a-b r+2 \lambda_{2} \sigma_{r}^{2}\right) f_{r}+\frac{1}{2} \sigma_{r}^{2} f_{r r}-\sigma_{r}^{2} \frac{f_{r}^{2}}{f}\right) \\
& -2 f(x-g)^{2}\left(g_{t}-r g+\left(a-b r+\lambda_{2} \sigma_{r}^{2}\right) g_{r}+\frac{1}{2} \sigma_{r}^{2} g_{r r}+u-\lambda_{1} v\right)=0 .
\end{aligned}
$$

In order to eliminate the dependence on $x$, we decompose (22) into the following two equations:

$$
\begin{aligned}
& f_{t}+\left(2 r-\lambda_{1}^{2}-\lambda_{2}^{2} \sigma_{r}^{2}\right) f+\left(a-b r+2 \lambda_{2} \sigma_{r}^{2}\right) f_{r}+\frac{1}{2} \sigma_{r}^{2} f_{r r}-\sigma_{r}^{2} \frac{f_{r}^{2}}{f}=0, \quad f(T, r)=1 ; \\
& g_{t}-r g+\left(a-b r+\lambda_{2} \sigma_{r}^{2}\right) g_{r}+\frac{1}{2} \sigma_{r}^{2} g_{r r}+u-\lambda_{1} v=0, \quad g(T, r)=\eta .
\end{aligned}
$$

Lemma 1 Assume that a solution to (23) is of the structure $f(t, r)=\mathrm{e}^{D_{1}(t)+D_{2}(t) r}$, with terminal conditions: $D_{1}(T)=0$ and $D_{2}(T)=0$, then we have the following conclusions:

(i) Under the condition of

$$
\begin{cases}\lambda_{2} \in \mathbb{R}, & b^{2}<4 k_{1}, \\ \lambda_{2} \neq \frac{b}{k_{1}}, & b^{2}=4 k_{1}, \\ \lambda_{2}<\xi_{1} \text { or } \lambda_{2}>\xi_{2}, & b^{2}>4 k_{1},\end{cases}
$$

then $D_{2}(t)$ and $D_{1}(t)$ are given by (a8) and (a10) in the Appendix respectively.

(ii) Under the condition of

$$
\begin{cases}\lambda_{2}=\frac{b}{k_{1}}, & b^{2}=4 k_{1}, \\ \lambda_{2}=\xi_{1} \text { or } \lambda_{2}=\xi_{2}, & b^{2}>4 k_{1},\end{cases}
$$

then $D_{2}(t)$ and $D_{1}(t)$ are given by (a13) and (a14) in the Appendix respectively.

(iii) Under the condition of $\xi_{1}<\lambda_{2}<\xi_{2}$ and $b^{2}>4 k_{1}$, then $D_{2}(t)$ and $D_{1}(t)$ are given by (a16) and (a17) in the Appendix respectively.

Here $\xi_{1}$ and $\xi_{2}$ are given by (a6) in the Appendix.

Proof See the Appendix (A1).

In order to solve (24), we have the following lemma.

Lemma 2 Suppose that

$$
g(t, r)=\left(u-\lambda_{1} v\right) \int_{t}^{T} \tilde{g}(s, r) \mathrm{d} s+\eta \tilde{g}(t, r)
$$

is a solution to (24), then $\tilde{g}(t, r)$ satisfies:

$$
\tilde{g}_{t}-r \tilde{g}+\left(a-b r+\lambda_{2} \sigma_{r}^{2}\right) \tilde{g}_{r}+\frac{1}{2} \sigma_{r}^{2} \tilde{g}_{r r}=0, \quad \tilde{g}(T, r)=1 .
$$


Proof See the Appendix (A2).

Lemma 3 Assume that a solution to $(25)$ is of the form $\tilde{g}(t, r)=\mathrm{e}^{D_{3}(t)+D_{4}(t) r}$, with terminal conditions: $D_{3}(T)=0$ and $D_{4}(T)=0$, then $D_{4}(t)$ and $D_{3}(t)$ are given by (a26) and (a28) in the Appendix respectively.

Proof See the Appendix (A3).

Further, we have

$$
\frac{H_{x}}{H_{x x}}=x-g, \quad \frac{H_{r x}}{H_{x x}}=D_{2}(t)(x-g)-g_{r} .
$$

As a result, considering $X(t)=x$ and $r(t)=r$, we draw the following conclusion.

Theorem 1 For given constants $\eta$ and $C$, the optimal investment strategies of the problems (9) and (11) are given by

$$
\begin{aligned}
\pi_{1}^{*}(t)= & -\frac{\lambda_{1}}{\sigma_{1}} \cdot(X(t)-g(t, r))+\frac{v}{\sigma_{1}}, \\
\pi_{2}^{*}(t)= & \frac{\sigma_{r}}{\sigma_{B}} \cdot \frac{\sigma_{2} \lambda_{1}-\sigma_{1} \lambda_{2}}{\sigma_{1}} \cdot(X(t)-g(t, r)) \\
& +\frac{\sigma_{r}}{\sigma_{B}} \cdot\left(D_{2}(t)(X(t)-g(t, r))-\frac{\partial g(t, r)}{\partial r}\right)-\frac{\sigma_{2}}{\sigma_{1}} \cdot \frac{\sigma_{r}}{\sigma_{B}} v,
\end{aligned}
$$

where

$$
\begin{aligned}
& g(t, r)=\left(u-\lambda_{1} v\right) \int_{t}^{T} \mathrm{e}^{D_{3}(s)+D_{4}(s) r} \mathrm{~d} s+\eta \mathrm{e}^{D_{3}(t)+D_{4}(t) r}, \\
& \frac{\partial g(t, r)}{\partial r}=\left(u-\lambda_{1} v\right) \int_{t}^{T} D_{4}(s) \mathrm{e}^{D_{3}(s)+D_{4}(s) r} \mathrm{~d} s+\eta D_{4}(t) \mathrm{e}^{D_{3}(t)+D_{4}(t) r},
\end{aligned}
$$

and $D_{1}(t), D_{2}(t), D_{3}(t)$ and $D_{4}(t)$ are given by Lemma 1 and Lemma 3 , respectively.

\section{The Efficient Frontier}

In this section, we will use Lagrange duality theorem to derive the explicit expression of the efficient frontier and provide some special cases.

According to (14), the minimized value of (11) is written as $f\left(0, r_{0}\right)\left(x_{0}-g\left(0, r_{0}\right)\right)^{2}$. So the minimized value of (9) is given by

$$
\begin{aligned}
& \bar{L}_{\min }\left(\pi_{1}^{*}(t), \pi_{2}^{*}(t), \eta\right) \\
= & f\left(0, r_{0}\right)\left(x_{0}-g\left(0, r_{0}\right)\right)^{2}-(C-\eta)^{2} \\
= & f\left(0, r_{0}\right)\left(x_{0}-\left(u-\lambda_{1} v\right) \int_{0}^{T} \tilde{g}(s, r(s)) \mathrm{d} s-\eta \tilde{g}\left(0, r_{0}\right)\right)^{2}-(C-\eta)^{2} \\
= & \left(f\left(0, r_{0}\right) \tilde{g}^{2}\left(0, r_{0}\right)-1\right) \eta^{2}-2 \eta\left(\psi f\left(0, r_{0}\right) \tilde{g}\left(0, r_{0}\right)-C\right)+\psi^{2} f\left(0, r_{0}\right)-C^{2},
\end{aligned}
$$

where

$$
\psi=x_{0}-\left(u-\lambda_{1} v\right) \int_{0}^{T} \tilde{g}(s, r(s)) \mathrm{d} s .
$$

Noting that

$$
f\left(0, r_{0}\right) \tilde{g}^{2}\left(0, r_{0}\right)-1=\mathrm{e}^{D_{1}(0)+2 D_{3}(0)+\left(D_{2}(0)+2 D_{4}(0)\right) r_{0}}-1,
$$

we have the following conclusion. 
Lemma $4 D_{2}(t), D_{4}(t)$ and $D_{1}(t)+2 D_{3}(t)$ increases with respect to the variable $t$. Moreover, we have $D_{2}(t)<0, D_{4}(t)<0$ and $D_{1}(t)+2 D_{3}(t)<0$, for $\forall t \in[0, T]$.

Proof See the Appendix (A4).

According to Lemma 4, we have

$$
f\left(0, r_{0}\right) \tilde{g}^{2}\left(0, r_{0}\right)-1=\mathrm{e}^{D_{1}(0)+2 D_{3}(0)+\left(D_{2}(0)+2 D_{4}(0)\right) r_{0}}-1<0 .
$$

Therefore, $\bar{L}_{\min }\left(\pi_{1}^{*}(t), \pi_{2}^{*}(t), \eta\right)$ can be maximized when $\eta$ is given by

$$
\eta^{*}=\frac{\psi f\left(0, r_{0}\right) \tilde{g}\left(0, r_{0}\right)-C}{\left(f\left(0, r_{0}\right) \tilde{g}^{2}\left(0, r_{0}\right)-1\right)}=\frac{\psi \mathrm{e}^{D_{1}(0)+D_{3}(0)+\left(D_{2}(0)+D_{4}(0)\right) r_{0}}-C}{\mathrm{e}^{D_{1}(0)+2 D_{3}(0)+\left(D_{2}(0)+2 D_{4}(0)\right) r_{0}}-1} .
$$

In addition, the maximized value of $\bar{L}_{\min }\left(\pi_{1}^{*}(t), \pi_{2}^{*}(t), \eta\right)$ is

$$
\bar{L}_{\max \min }\left(\pi_{1}^{*}(t), \pi_{2}^{*}(t), \eta^{*}\right)=\frac{f\left(0, r_{0}\right) \tilde{g}^{2}\left(0, r_{0}\right)}{1-f\left(0, r_{0}\right) \tilde{g}^{2}\left(0, r_{0}\right)}\left(C-\psi \tilde{g}^{-1}\left(0, r_{0}\right)\right)^{2} .
$$

In conclusion, we summarize the above results in the following proposition.

Theorem 2 For a given constant $C \in \mathbb{R}$, the efficient strategies for the original meanvariance problem (8) corresponding to $E(X(T))=C$ are given by

$$
\begin{aligned}
\pi_{1}^{*}(t)= & -\frac{\lambda_{1}}{\sigma_{1}} \cdot(X(t)-g(t, r))+\frac{v}{\sigma_{1}} \\
\pi_{2}^{*}(t)= & \frac{\sigma_{r}}{\sigma_{B}} \cdot \frac{\sigma_{2} \lambda_{1}-\sigma_{1} \lambda_{2}}{\sigma_{1}} \cdot(X(t)-g(t, r)) \\
& +\frac{\sigma_{r}}{\sigma_{B}} \cdot\left(D_{2}(t)(X(t)-g(t, r))-\frac{\partial g(t, r)}{\partial r}\right)-\frac{\sigma_{2}}{\sigma_{1}} \cdot \frac{\sigma_{r}}{\sigma_{B}} v
\end{aligned}
$$

with the efficient frontier given by

$$
\operatorname{Var} X(T)=\frac{1}{\mathrm{e}^{-D_{1}(0)-2 D_{3}(0)-\left(D_{2}(0)+2 D_{4}(0)\right) r_{0}}-1}\left(E(X(T))-\psi \mathrm{e}^{-D_{3}(0)-D_{4}(0) r_{0}}\right)^{2},
$$

where

$$
\begin{aligned}
& \psi=x_{0}-\left(u-\lambda_{1} v\right) \int_{0}^{T} \tilde{g}(s, r) \mathrm{d} s, \quad \eta^{*}=\frac{\psi \mathrm{e}^{D_{1}(0)+D_{3}(0)+\left(D_{2}(0)+D_{4}(0)\right) r_{0}}-C}{\mathrm{e}^{D_{1}(0)+2 D_{3}(0)+\left(D_{2}(0)+2 D_{4}(0)\right) r_{0}-1}} \\
& g(t, r)=\left(u-\lambda_{1} v\right) \int_{t}^{T} \mathrm{e}^{D_{3}(s)+D_{4}(s) r} \mathrm{~d} s+\eta^{*} \mathrm{e}^{D_{3}(t)+D_{4}(t) r} \\
& \frac{\partial g(t, r)}{\partial r}=\left(u-\lambda_{1} v\right) \int_{t}^{T} D_{4}(s) \mathrm{e}^{D_{3}(s)+D_{4}(s) r} \mathrm{~d} s+\eta^{*} D_{4}(t) \mathrm{e}^{D_{3}(t)+D_{4}(t) r}
\end{aligned}
$$

Here, $D_{1}(t), D_{2}(t), D_{3}(t)$ and $D_{4}(t)$ are given by Lemma 1 and Lemma 3, respectively.

Remark 2 From Theorem 3, some conclusions are drawn as follows. (i) $\pi_{1}^{*}(t)$ is correlated with the parameters $a, b, k_{1}, k_{2}, \lambda_{1}, \sigma_{1}, \lambda_{2}, u, v$, but it is not correlated with the parameter $\sigma_{2}$. Although the parameter $\sigma_{2}$ has effect on the dynamics of stock price, $\pi_{1}^{*}(t)$ doesn't depend on $\sigma_{2}$. It is greatly surprised us. (ii) $\pi_{2}^{*}(t)$ depends on all the parameters $a, b, k_{1}, k_{2}, \lambda_{1}, \sigma_{1}, \lambda_{2}$, $u, v$ and $\sigma_{2}$. Although the dynamics of the zero-coupon bond and interest rate are driven by the same Brownian motion, $\pi_{2}^{*}(t)$ is still affected by the parameters of stock price. It also 
surprised us. (iii) The efficient frontier (36) depends on the parameters $a, b, k_{1}, k_{2}, \lambda_{1}, \lambda_{2}, u, v$, but doesn't depend on $\sigma_{1}$ and $\sigma_{2}$. We can find from the dynamic equation of stock price that $\sigma_{1}$ and $\sigma_{2}$ represent the volatility resulted from the two different Brownian motions respectively, whereas the investors' risks measured by $\operatorname{Var} X(T)$ don't depend on the volatility of the stock. This implies that it is very necessary for us to analyze the sensitivity of model parameters on the efficient policies and the efficient frontier.

Remark 3 From (34)-(36), we can draw the following some conclusions. (i) The efficient policies and the efficient frontier are not deterministic functions as well, but are dynamic functions. They depend on the state of short rate $r(t)$ and are affected by all the parameters of the liability and interest rate. (ii) The efficient frontier in the mean-standard deviation diagram is still a straight line, no matter at which state interest rate is. To be specific, let $\sigma[X(T)]$ be the standard deviation of the terminal surplus, then (36) gives

$$
E(X(T))=\psi \mathrm{e}^{-D_{3}(0)-D_{4}(0) r_{0}}+\sigma[X(T)] \sqrt{\mathrm{e}^{-D_{1}(0)-2 D_{3}(0)-\left(D_{2}(0)+2 D_{4}(0)\right) r_{0}-1}},
$$

which is also called the capital market line in the portfolio theory.

Remark 4 When $\sigma[X(T)]=0$, then we have $E(X(T))=\psi \mathrm{e}^{-D_{3}(0)-D_{4}(0) r_{0}}$. Therefore, when $C$ runs over $\left[\psi \mathrm{e}^{-D_{3}(0)-D_{4}(0) r_{0}},+\infty\right)$, the efficient frontier is composed of all the points $(C, \operatorname{Var} X(T))$.

In order to compare the conclusion from Theorem 3 with those in the existing literatures, we give the following three special cases.

Special case 1 If there is no liability, i.e., $u=v=0$, then we get

$$
\psi=x_{0}, \quad \eta^{*}=\frac{x_{0} \mathrm{e}^{D_{1}(0)+D_{3}(0)+\left(D_{2}(0)+D_{4}(0)\right) r_{0}}-C}{\mathrm{e}^{D_{1}(0)+2 D_{3}(0)+\left(D_{2}(0)+2 D_{4}(0)\right) r_{0}}-1}, \quad g(t, r(t))=\eta^{*} \mathrm{e}^{D_{3}(t)+D_{4}(t) r(t)} .
$$

Therefore the efficient strategies for the original mean-variance problem (8) are given by

$$
\begin{aligned}
\pi_{1}^{*}(t)= & -\frac{\lambda_{1}}{\sigma_{1}} \cdot\left(X(t)-\eta^{*} \mathrm{e}^{D_{3}(t)+D_{4}(t) r(t)}\right), \\
\pi_{2}^{*}(t)= & \frac{\sigma_{r}}{\sigma_{B}} \cdot \frac{\sigma_{2} \lambda_{1}-\sigma_{1} \lambda_{2}}{\sigma_{1}} \cdot\left(X(t)-\eta^{*} \mathrm{e}^{D_{3}(t)+D_{4}(t) r(t)}\right) \\
& +\frac{\sigma_{r}}{\sigma_{B}} \cdot\left(D_{2}(t)\left(X(t)-\eta^{*} \mathrm{e}^{D_{3}(t)+D_{4}(t) r(t)}\right)-\frac{\partial g(t, r)}{\partial r}\right),
\end{aligned}
$$

with the efficient frontier given by

$$
\operatorname{Var} X(T)=\frac{1}{\mathrm{e}^{-D_{1}(0)-2 D_{3}(0)-\left(D_{2}(0)+2 D_{4}(0)\right) r_{0}}-1}\left(E(X(T))-x_{0} \mathrm{e}^{-D_{3}(0)-D_{4}(0) r_{0}}\right)^{2},
$$

where $D_{1}(t), D_{2}(t), D_{3}(t)$ and $D_{4}(t)$ are still given by Lemma 1 and Lemma 3 , respectively.

Remark 5 When $u=v=0$, our model is reduced to that studied by Ferland and Watier ${ }^{[7]}$, who used backward stochastic differential equation (BSDE) theory to investigate a dynamic mean-variance portfolio choice problem in the CIR framework. Therefore, our work extends the conclusion obtained by Ferland and Watier ${ }^{[7]}$.

Special case 2 If interest rate is a constant, i.e., $a=b=k_{1}=k_{2}=0$, then the zerocoupon bond is reduced to one risk-free asset. Therefore, the zero-coupon bond should not be 
considered in the investment process such that $\pi_{2}^{*}(t)=0$. In addition, we obtain

$$
D_{1}(t)=-\lambda_{1}^{2}(T-t), \quad D_{2}(t)=2(T-t), \quad D_{3}(t)=0, \quad D_{4}(t)=-(T-t) .
$$

And it leads to that

$$
\begin{aligned}
& \psi=x_{0}-\left(u-\lambda_{1} v\right) \int_{0}^{T} \mathrm{e}^{-(T-s) r(s)} \mathrm{d} s, \quad \eta^{*}=\frac{\psi \mathrm{e}^{\left(-\lambda_{1}^{2}+r_{0}\right) T}-C}{\mathrm{e}^{-\lambda_{1}^{2} T}-1}, \\
& g(t, r)=\left(u-\lambda_{1} v\right) \int_{t}^{T} \mathrm{e}^{-(T-s) r(s)} \mathrm{d} s+\eta^{*} \mathrm{e}^{-(T-t) r(t)} .
\end{aligned}
$$

Finally, the efficient policies and the efficient frontier for the original mean-variance problem (8) are respectively given by

$$
\begin{aligned}
& \pi_{1}^{*}(t)=-\frac{\lambda_{1}}{\sigma_{1}} \cdot\left(X(t)-\left(u-\lambda_{1} v\right) \int_{t}^{T} \mathrm{e}^{-(T-s) r(s)} \mathrm{d} s-\eta^{*} \mathrm{e}^{-(T-t) r(t)}\right)+\frac{v}{\sigma_{1}} \\
& \operatorname{Var} X(T)=\frac{1}{\mathrm{e}^{\lambda_{1}^{2} T}-1}\left(E(X(T))-\left(x_{0}-\left(u-\lambda_{1} v\right) \int_{0}^{T} \mathrm{e}^{-(T-s) r(s)} \mathrm{d} s\right) \mathrm{e}^{(T-t) r_{0}}\right)^{2} .
\end{aligned}
$$

Remark 6 It can be easily seen that (47) and (48) coincide with the conclusions derived by Xie, et al. ${ }^{[12]}$ when liability dynamics is completely positive correlation with stock price dynamics. It shows that our work extends the model of Xie, et al. ${ }^{[12]}$ to stochastic affine interest rate environment, and successfully solves the dynamic mean-variance portfolio choice problem with stochastic interest rate.

Special case 3 If interest rate is reduced to be constant and liability is not considered, i.e., $u=v=0$ and $a=b=k_{1}=k_{2}=0$, then we have $\pi_{2}^{*}(t)=0$, and

$$
\psi=x_{0}, \quad \eta^{*}=\frac{x_{0} \mathrm{e}^{\left(-\lambda_{1}^{2}+r_{0}\right) T}-C}{\mathrm{e}^{-\lambda_{1}^{2} T}-1} .
$$

As a result, the efficient strategies and the efficient frontier for the original mean-variance problem (8) are respectively given by

$$
\begin{aligned}
& \pi_{1}^{*}(t)=-\frac{\lambda_{1}}{\sigma_{1}} \cdot\left(X(t)+\eta^{*} \mathrm{e}^{-(T-t) r(t)}\right), \\
& \operatorname{Var} X(T)=\frac{1}{\mathrm{e}^{\lambda_{1}^{2} T}-1}\left(E(X(T))-x_{0} \mathrm{e}^{(T-t) r_{0}}\right)^{2} .
\end{aligned}
$$

Remark 7 Notice that (50) and (51) are consistent with the conclusions obtained by Zhou and $\mathrm{Li}^{[4]}$. It implies that our work is the most important extension of the model studied by Zhou and $\mathrm{Li}^{[4]}$.

\section{Numerical Illustrations}

In this section, we provide a numerical example to illustrate the impact of the parameters of interest rate and liability on the efficient strategy and the efficient frontier. In the following numerical illustrations, we focus on the case of $\Delta_{1}>0$ and $k_{1} \lambda_{2}^{2}>2$, i.e., $\lambda_{2} \in \mathbb{R}, b^{2}<4 k_{1}$ and $k_{1} \lambda_{2}^{2}>2$. Under these conditions, $D_{1}(t), D_{2}(t), D_{3}(t)$ and $D_{4}(t)$ are given by (a10), (a8), 
(a28) and (a26) in the Appendix respectively. The analysis in the other cases is similar to that in the case of $\lambda_{2} \in \mathbb{R}, b^{2}<4 k_{1}$ and $k_{1} \lambda_{2}^{2}>2$. Therefore, we assume that the main parameters are given by $a=0.018712, b=0.2339, k_{1}=0.51, k_{2}=0.5, r(0)=0.05, \sigma_{1}=0.2, \lambda_{1}=0.2$, $\sigma_{2}=0.02, \lambda_{2}=2, u=0.07, v=0.22, t=0, T=1, X(0)=100$.

Note that the values of $a$ and $b$ above mentioned come from the work of Deelstra, et al. ${ }^{[17]}$. In the following analysis we denote the optimal amount invested in the cash by $\pi_{0}^{*}(t)$, denote the optimal amount invested in the stock by $\pi_{1}^{*}(t)$, and denote the optimal amount in the zero-coupon bond by $\pi_{2}^{*}(t)$.

\subsection{Sensitivity Analysis on the Efficient Strategy}

From Figures 1 6, we can draw the following some instructive conclusions.

(a1) $\pi_{0}^{*}(t)$ decreases with respect to (w.r.t) the parameter $b$, while $\pi_{1}^{*}(t)$ and $\pi_{2}^{*}(t)$ increase w.r.t the parameter $b$. In fact, we can see that the expected value of interest rate becomes smaller as the value of $b$ becomes larger. A smaller value of the expected interest rate leads to a less amount invested in the cash. Accordingly, the amount invested in the stock and zero-coupon bond will increase. Therefore, this conclusion is consistent with the economic implication of $b$.

(a2) $\pi_{0}^{*}(t)$ and $\pi_{2}^{*}(t)$ are decreasing functions of the parameter $k_{1}$, while $\pi_{1}^{*}(t)$ is increasing in $k_{1}$. As matter of fact, the larger the value of $k_{1}$, the larger interest rate risk, which will lead to the less amount invested in the cash and zero-coupon bond. On the contrary, the amount in the stock will be increasing. This conclusion conforms to our intuition.

(a3) The trend between $\pi_{0}^{*}(t)$ and the parameter $\lambda_{1}$ is same, while the trend between $\pi_{1}^{*}(t)$ and $\lambda_{1}$ is opposite, $\pi_{2}^{*}(t)$ almost remain unchanged in $\lambda_{1}$. It displays that the amount in the cash is increasing as the value of $\lambda_{1}$ is increasing, while the amount invested in the stock is decreasing. Meantime, $\lambda_{1}$ almost has no effect on the amount in the zero-coupon bond.

(a4) $\pi_{0}^{*}(t)$ increases w.r.t the parameter $\lambda_{2}, \pi_{1}^{*}(t)$ almost remains unchanged in $\lambda_{2}$, while $\pi_{2}^{*}(t)$ is a decreasing function in $\lambda_{2}$. It tells us that we should invest more money in the cash when the value of $\lambda_{2}$ becomes bigger, while invest less money in the zero-coupon bond. What surprised us is that $\lambda_{2}$ almost has no impact on the amount in the stock. We should keep this conclusion in mind in the practice of investment.

(a5) $\pi_{0}^{*}(t)$ is decreasing in $u$, while $\pi_{1}^{*}(t)$ and $\pi_{2}^{*}(t)$ are increasing in $u$. In reality, the bigger the value of $u$, the bigger the expected liability. In order to hedge the risk resulted from random liability, investors need increasing the amount in the risky assets. It is obvious that the amount invested in the risk-free asset is reduced.

(a6) $\pi_{0}^{*}(t)$ and $\pi_{2}^{*}(t)$ have decreasing trends in the parameter $v$, while $\pi_{1}^{*}(t)$ has an increasing trend in $v$. A larger value of $v$ will cause a bigger volatility risk. It implies that investors need increasing the amount in the stock, while decreasing the amount in the cash and zero-coupon bond.

\subsection{Sensitivity Analysis on the Efficient Frontier}

Figures $7 \sim 12$ illustrate the relationships between the standard deviation $\sigma[X(T)]$ and the expected return $E(X(T))=C$. According to our observation on Figure 4 and Figure 5, we obtain the following some instructive conclusions: 


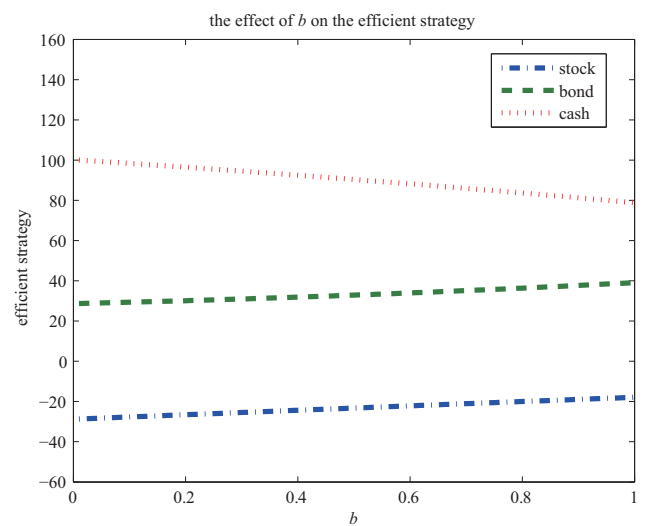

Figure 1 The effect of $b$ on the efficient strategy

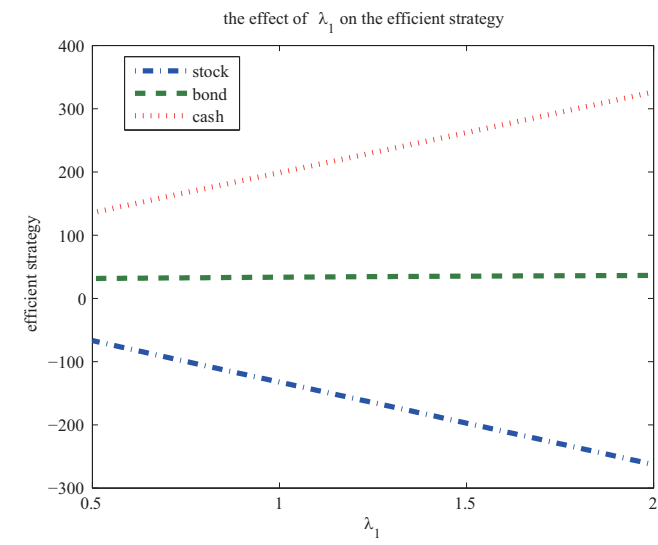

Figure 3 The effect of $\lambda_{1}$ on the efficient strategy

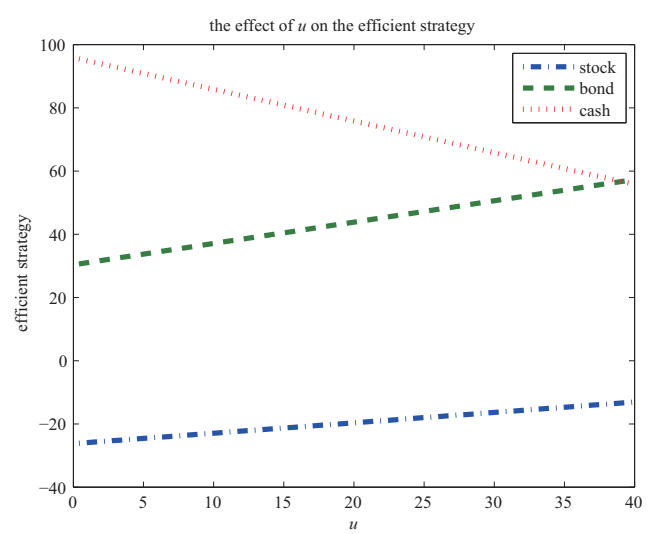

Figure 5 The effect of $u$ on the efficient strategy

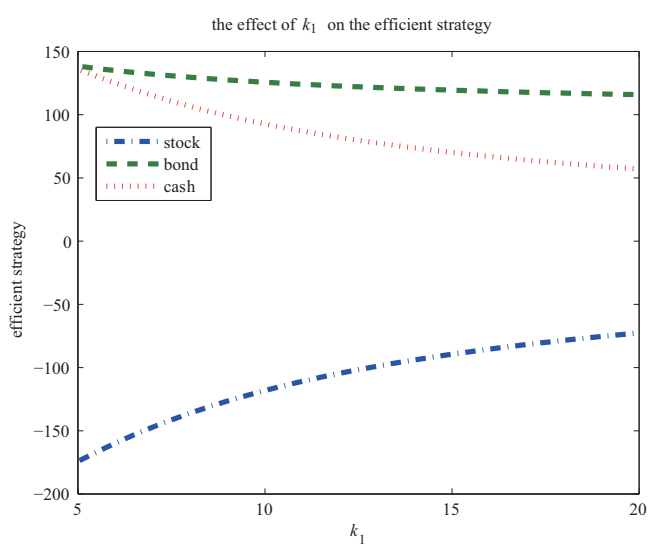

Figure 2 The effect of $k_{1}$ on the efficient strategy

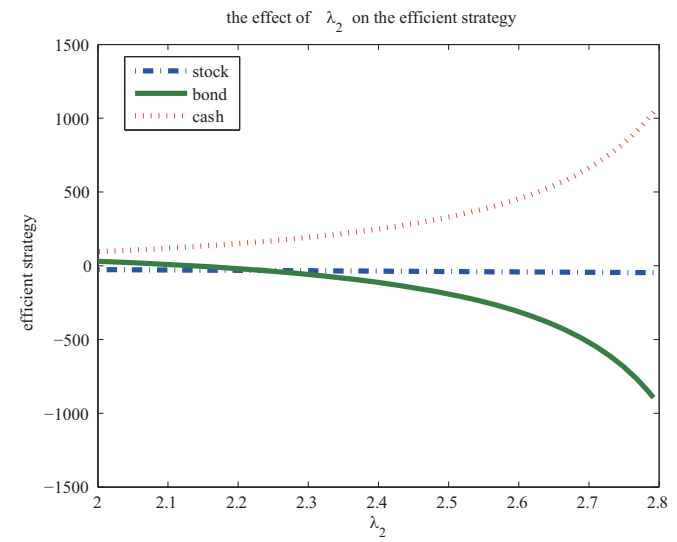

Figure 4 The effect of $\lambda_{2}$ on the efficient strategy

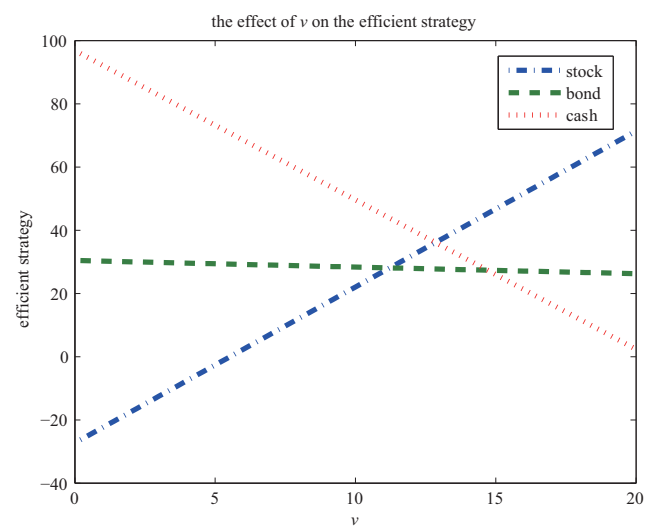

Figure 6 The effect of $v$ on the efficient strategy 

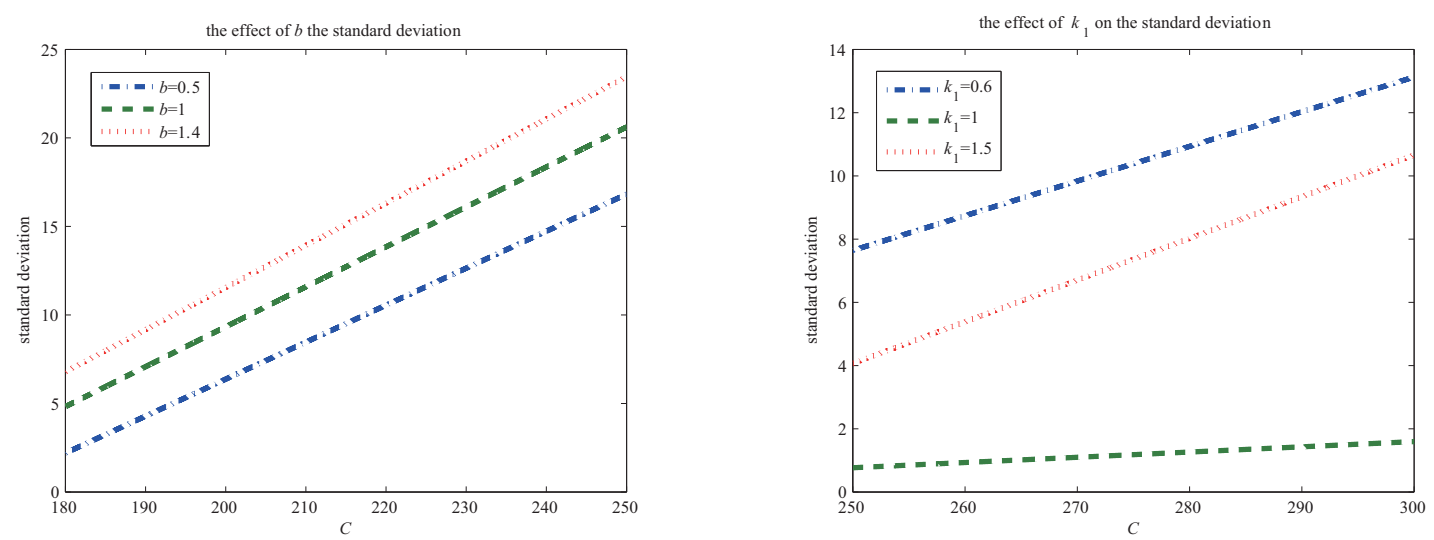

Figure 7 The effect of $b$ on the standard deviation

Figure 8 The effect of $k_{1}$ on the standard deviation
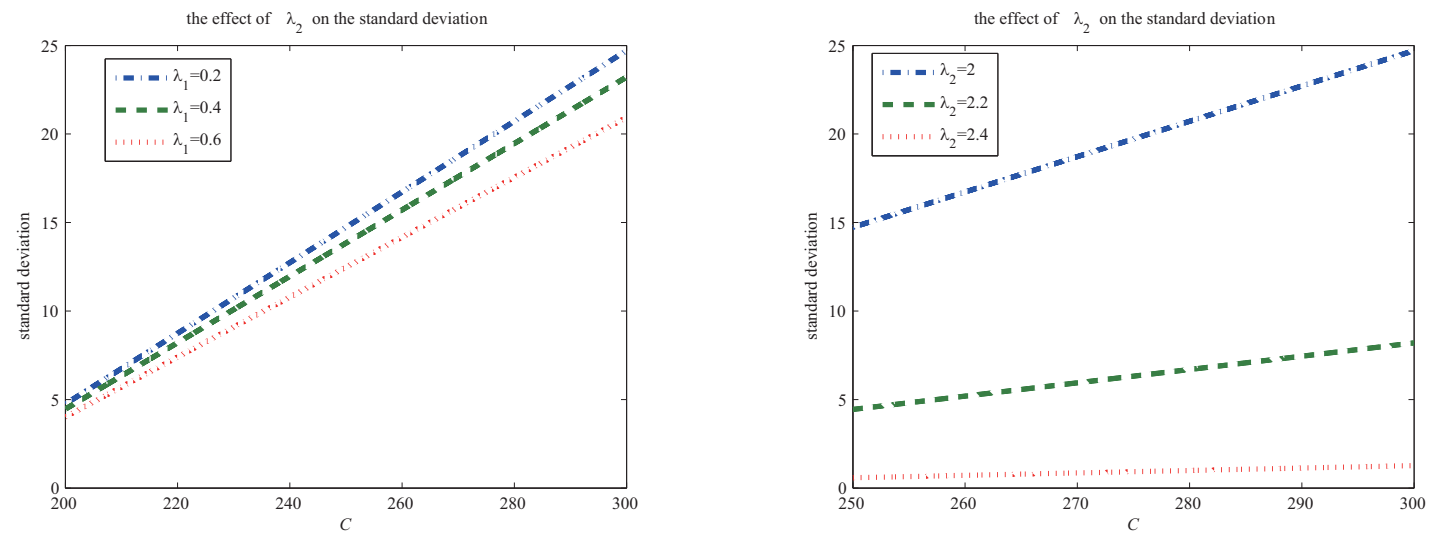

Figure 9 The effect of $\lambda_{1}$ on the standard deviation Figure 10 The effect of $\lambda_{2}$ on the standard deviation
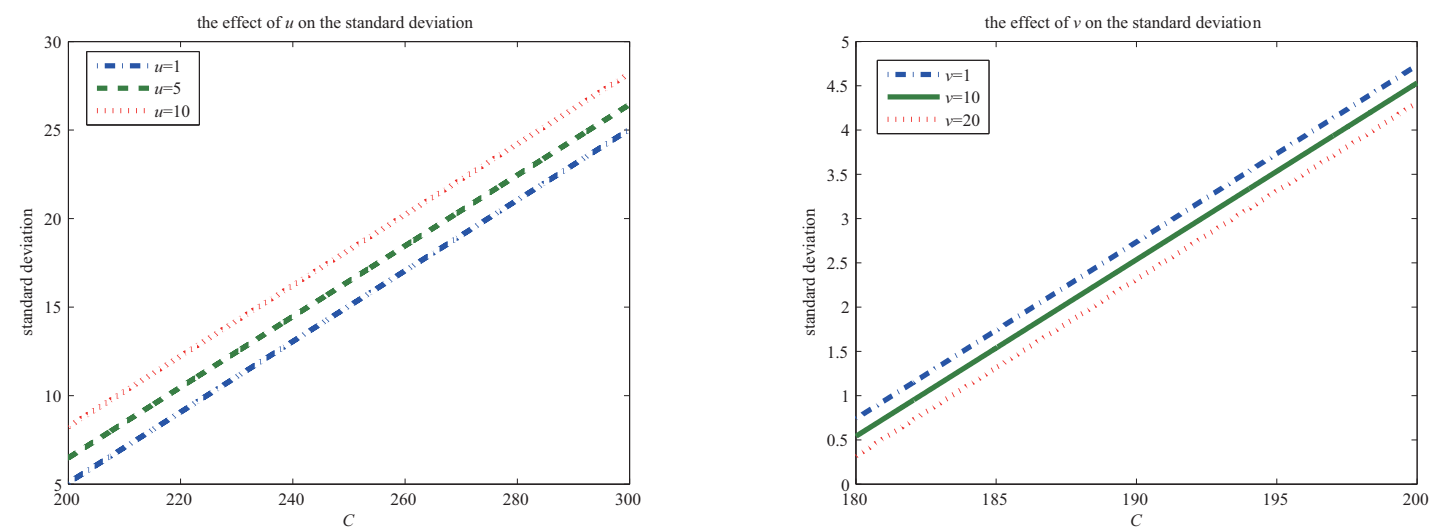

Figure 11 The effect of $u$ on the standard deviation Figure 12 The effect of $v$ on the standard deviation 
(b1) $\sigma[X(T)]$ increases w.r.t the parameter $b$ for a given expected return. From the points of economic implication of $b$, a larger value of $b$ will lead to a greater amount in the stock and zero-coupon bond and a smaller amount in the cash. Therefore, investment yields will greatly increase. The principle of capital market line tells us that investors should undertake more risks.

(b2) $\sigma[X(T)]$ first decreases then increases w.r.t. the parameter $k_{1}$ for a given expected return. This conclusion tells us that we need to pay close attention to the effect of $k_{1}$ on the risk level.

(b3) $\sigma[X(T)]$ is a decreasing function in the parameter $\lambda_{1}$. According to (a3) in the previous subsection, the total wealth of investors is decreasing when the value of $\lambda_{1}$ is increasing. The less total wealth, the less the risks taken on. Therefore, $\sigma[X(T)]$ measuring the risks is decreasing. This conclusion conforms to the principle of capital market line.

(b4) $\sigma[X(T)]$ has a decreasing trend as the value of $\lambda_{2}$ rises under the same expected wealth level. From (a4) in the previous subsection, we find that the risk resulted from stocks remains unchanged and the risk from zero-coupon bond decreases all the time when the value of $\lambda_{2}$ increases. Therefore, we draw the conclusion that the comprehensive risks from financial market will decrease.

(b5) $\sigma[X(T)]$ has the same trend as the parameter $u$ for a fixed level of expected return. From the economic implications of $u$, which represents the expected level of liability. It means that the larger the value of $u$, the larger the value of liability. In order to hedge the risk caused by the liability, investors need to hold more shares of stocks and zero-coupon bonds. At the same time, it requires investors to take on more risks.

(b6) $\sigma[X(T)]$ decreases w.r.t. the parameter $v$. Although a larger value of $v$ leads to the more amount in the stock and the less amount in the cash and zero-coupon bond, the total risks investors need to take on is decreasing. We should keep this conclusion in mind.

\section{Conclusions}

Liability factor is often faced with in the investment activities. This paper takes liability factor into consideration and focuses on a dynamic mean-variance portfolio selection problem with random liability in an affine interest rate environment. Applying dynamic programming principle and Lagrange duality theorem, we obtain the explicit expressions of the efficient policies and the efficient frontier. Finally, a numerical example is provided to analyze the dynamic behavior of the efficient policies and the efficient frontier. Our model extends the works of Ferland and Watier ${ }^{[7]}$, Xie, et al. ${ }^{[12]}$, Korn and $\mathrm{Kraft}^{[16]}$, and Gao ${ }^{[18]}$. Some interesting and instructive main conclusions are found as follows: (i) Although the parameter $\sigma_{2}$ has impacted on the dynamics of stock price, $\pi_{1}^{*}(t)$ doesn't depend on $\sigma_{2}$; (ii) $\pi_{2}^{*}(t)$ not only depends on the parameters of zero-coupon bond but also depends on the parameters of stock price and liability process; (iii) The efficient frontier in the mean-standard deviation diagram is still a straight line, no matter at which state interest rate is.

As far as we know, there is no work on the dynamic mean-variance portfolio choice problem with stochastic affine interest rate model. In addition, we have investigated affine interest rate model and considered random liability factor and successfully obtained the closed-form solutions 
to the efficient policies and the efficient frontier. However, our model has also some limits: (i) The liability process is the simplest model and without jump; (ii) We only consider stochastic affine interest rate model and doesn't consider stochastic volatility model, for example, the Heston model; (iii) Mean-variance framework is standard but is not the best criterion, and we can studied the problem under the other risk criterion, for example, the VaR (value at risk) constraint and down-side risk minimization criterion. In future research, we will devote ourselves to studying these problems.

\section{References}

[1] Markowitz H. Portfolio selection. Journal of Finance, 1952, 7(1): 77-91.

[2] Bajeux-Besnainou I, Portait R. Dynamic asset allocation in a mean-variance framework. Management Science, 1998, 44(11): S79-S95.

[3] Li D, Ng W L. Optimal dynamic portfolio selection: Multi-period mean-variance formulation. Mathematical Finance, 2000, 10(3): 387-406.

[4] Zhou X Y, Li D. Continuous time mean-variance portfolio selection: A stochastic LQ framework. Applied Mathematics and Optimization, 2000, 42(1): 19-33.

[5] Fu C, Lari-Lavassani A, Li X. Dynamic mean-variance portfolio selection with borrowing constraint. European Journal of Operational Research, 2010, 200(1): 312-319.

[6] Lim A E B, Zhou X Y. Mean-variance portfolio selection with random parameters in a complete market. Mathematics of Operations Research, 2002, 27(1): 101-120.

[7] Ferland R, Watier F. Mean-variance efficiency with extended CIR interest rates. Applied Stochastic Models in Business and Industry, 2010, 26(1): 71-84.

[8] Yi L, Li Z F, Li D. Multi-period portfolio selection for asset-liability management with uncertain investment horizon. Journal of Industrial and Management Optimization, 2008, 4(3): 535-552.

[9] Chen P, Yang H L. Markowitz's mean-variance asset-liability management with regime switching: A multi-period model. Applied Mathematical Finance, 2011, 18(1): 29-50.

[10] Yao H X, Zeng Y, Chen S M. Multi-period mean-variance asset-liability management with uncontrolled cash flow and uncertain time-horizon. Economic Modelling, 2013, 30(1): 492-500.

[11] Leippold M, Trojani F, Vanini P. Multi-period mean-variance efficient portfolios with endogenous liabilities. Quantitative Finance, 2011, 11(10): 1535-1546.

[12] Xie S X, Li Z F, Wang S Y. Continuous-time portfolio selection with liability: Mean-variance model and stochastic LQ approach. Insurance: Mathematics and Economics, 2008, 42(3): 943-953.

[13] Zeng Y, Li Z F. Asset-liability management under benchmark and mean-variance criteria in a jump diffusion market. Journal of Systems Science and Complexity, 2011, 24(2): 317-327.

[14] Chen P, Yang H L, Yin G. Markowitz's mean-variance asset-liability management with regime switching: A continuous-time model. Insurance: Mathematics and Economics, 2008, 43(3): 456-465.

[15] Yao H X, Lai Y Z, Li Y. Continuous-time mean-variance asset-liability management with endogenous liabilities. Insurance: Mathematics and Economics, 2013, 52(1): 6-17.

[16] Korn R, Kraft H. A stochastic control approach to portfolio problems with stochastic interest rates. SIAM Journal of Control and optimization, 2001, 40(4): 1250-1269.

[17] Deelstra G, Grasselli M, Koehl P F. Optimal investment strategies in the presence of a minimum guarantee. Insurance: Mathematics and Economics, 2003, 33(1): 189-207.

[18] Gao J W. Stochastic optimal control of DC pension funds. Insurance: Mathematics and Economics, 2008, 42(3): 1159-1164.

[19] Chang H, Chang K, Lu J M. Portfolio selection with liability and affine interest rate in the HARA utility framework. Abstract and Applied Analysis, 2014, http://dx.doi.org/10.1155/2014/312640.

[20] Li J Z, Wu R. Optimal investment problem with stochastic interest rate and stochastic volatility: Maximizing a power utility. Applied Stochastic Models in Business and Industry, 2009, 25(3): 407-420.

[21] Liu J. Portfolio selection in stochastic environments. The Review of Financial Studies, 2007, 20(1): 1-39.

[22] Chang H, Rong X M. An investment and consumption problem with CIR interest rate and stochastic volatility. Abstract and Applied Analysis, 2013, http://dx.doi.org/10.1155/2013/219397. 
[23] Guan G H, Liang Z X. Optimal reinsurance and investment strategies for insurer under interest rate and inflation risks. Insurance: Mathematics and Economics, 2014, 55(1): 105-115.

[24] Guan G H, Liang Z X. Optimal management of DC pension plan in a stochastic interest rate and stochastic volatility framework. Insurance: Mathematics and Economics, 2014, 57(1): 58-66.

[25] Vasicek O A. An equilibrium characterization of the term structure. Journal of Financial Economics, 1977, 5(2): 177-188.

[26] Cox J C, Ingersoll J E, Ross S A. A theory of the term structure of interest rates. Econometrica, 1985, 53(2): 385-408.

[27] Fleming W H, Soner H M. Controlled Markov processes and viscosity solutions. Springer-Verlag, New York, 1993.

\section{Appendix}

(A1) The proof of Lemma 1 Introducing $f(t, r)=\mathrm{e}^{D_{1}(t)+D_{2}(t) r}$ into (23) yields

$$
\begin{aligned}
& \mathrm{e}^{D_{1}(t)+D_{2}(t) r}\left\{\dot{D}_{1}(t)+\left(a+2 \lambda_{2} k_{2}\right) D_{2}(t)-\frac{1}{2} k_{2} D_{2}^{2}(t)-\lambda_{1}^{2}-k_{2} \lambda_{2}^{2}\right. \\
& \left.+r\left(\dot{D}_{2}(t)+2-k_{1} \lambda_{2}^{2}+\left(2 k_{1} \lambda_{2}-b\right) D_{2}(t)-\frac{1}{2} k_{1} D_{2}^{2}(t)\right)\right\}=0 .
\end{aligned}
$$

Comparing the coefficients on the both sides of (a1), we get

$$
\begin{array}{ll}
\dot{D}_{2}(t)+2-k_{1} \lambda_{2}^{2}+\left(2 k_{1} \lambda_{2}-b\right) D_{2}(t)-\frac{1}{2} k_{1} D_{2}^{2}(t)=0, & D_{2}(T)=0 \\
\dot{D}_{1}(t)+\left(a+2 \lambda_{2} k_{2}\right) D_{2}(t)-\frac{1}{2} k_{2} D_{2}^{2}(t)-\lambda_{1}^{2}-k_{2} \lambda_{2}^{2}=0, & D_{1}(T)=0 .
\end{array}
$$

The discriminant $\Delta_{1}$ for the quadratic equation

$$
\frac{1}{2} k_{1} D_{2}^{2}(t)-\left(2 k_{1} \lambda_{2}-b\right) D_{2}(t)-\left(2-k_{1} \lambda_{2}^{2}\right)=0
$$

is given by $\Delta_{1}=\left(2 k_{1} \lambda_{2}-b\right)^{2}+2 k_{1}\left(2-k_{1} \lambda_{2}^{2}\right)$. It is very obvious that (a4) has different roots depending on whether $\Delta_{1}>0, \Delta_{1}=0$ or $\Delta_{1}<0$. These cases will be respectively discussed as follows.

(i) If $\Delta_{1}>0$, i.e.,

$$
\left(2 k_{1} \lambda_{2}-b\right)^{2}+2 k_{1}\left(2-k_{1} \lambda_{2}^{2}\right)>0 \Rightarrow \begin{cases}\lambda_{2} \in \mathbb{R}, & b^{2}<4 k_{1}, \\ \lambda_{2} \neq \frac{b}{k_{1}}, & b^{2}=4 k_{1}, \\ \lambda_{2}<\xi_{1} \text { or } \lambda_{2}>\xi_{2}, & b^{2}>4 k_{1},\end{cases}
$$

where

$$
\xi_{1}=\frac{b}{k_{1}}-\frac{\sqrt{2 b^{2}-8 k_{1}}}{2 k_{1}}, \quad \xi_{2}=\frac{b}{k_{1}}+\frac{\sqrt{2 b^{2}-8 k_{1}}}{2 k_{1}},
$$

then (a4) has two different real roots, which are respectively given by

$$
m_{1,2}=\frac{2 k_{1} \lambda_{2}-b}{k_{1}} \pm \frac{\sqrt{\Delta_{1}}}{k_{1}} .
$$

Therefore (a2) can be rewritten as

$$
\begin{aligned}
& \dot{D}_{2}(t)=\frac{1}{2} k_{1}\left(D_{2}(t)-m_{1}\right)\left(D_{2}(t)-m_{2}\right) \\
& \Rightarrow \frac{1}{m_{1}-m_{2}} \int_{t}^{T}\left(\frac{1}{D_{2}(t)-m_{1}}-\frac{1}{D_{2}(t)-m_{2}}\right) \mathrm{d} D_{2}(t)=\frac{1}{2} k_{1}(T-t)
\end{aligned}
$$




$$
\Rightarrow D_{2}(t)=\frac{m_{1} m_{2}\left(1-\exp \left\{\frac{1}{2} k_{1}\left(m_{1}-m_{2}\right)(T-t)\right\}\right)}{m_{1}-m_{2} \exp \left\{\frac{1}{2} k_{1}\left(m_{1}-m_{2}\right)(T-t)\right\}} .
$$

Using (a2) $\times k_{2}-(\mathrm{a} 3) \times k_{1}$, we obtain

$$
\dot{D}_{1}(t)=\frac{k_{2}}{k_{1}} \dot{D}_{2}(t)-\frac{a k_{1}+b k_{2}}{k_{1}} D_{2}(t)+\frac{k_{1} \lambda_{1}^{2}+2 k_{2}}{k_{1}} .
$$

Solving the equation (a9), we derive

$$
\begin{aligned}
D_{1}(t)= & \frac{k_{2}}{k_{1}} D_{2}(t)-\frac{k_{1} \lambda_{1}^{2}+2 k_{2}}{k_{1}}(T-t) \\
& +\frac{a k_{1}+b k_{2}}{k_{1}}\left(m_{2}(T-t)-\frac{2}{k_{1}} \ln \frac{m_{1}-m_{2}}{m_{1}-m_{2} \exp \left\{\frac{1}{2} k_{1}\left(m_{1}-m_{2}\right)(T-t)\right\}}\right) .
\end{aligned}
$$

(ii) If $\Delta_{1}=0$, i.e.,

$$
\left(2 k_{1} \lambda_{2}-b\right)^{2}+2 k_{1}\left(2-k_{1} \lambda_{2}^{2}\right)=0 \Rightarrow \begin{cases}\lambda_{2}=\frac{b}{k_{1}}, & b^{2}=4 k_{1}, \\ \lambda_{2}=\xi_{1} \text { or } \lambda_{2}=\xi_{2}, & b^{2}>4 k_{1},\end{cases}
$$

then (a4) has the unique real root, which is given by

$$
\begin{aligned}
m_{3}=\frac{2 k_{1} \lambda_{2}-b}{k_{1}} . & \\
\dot{D}_{2}(t)=\frac{1}{2} k_{1}\left(D_{2}(t)-m_{3}\right)^{2} & \Rightarrow \frac{1}{\left(D_{2}(t)-m_{3}\right)^{2}} \dot{D}_{2}(t)=\frac{1}{2} k_{1} \\
& \Rightarrow D_{2}(t)=\frac{k_{1} m_{3}^{2}(T-t)}{k_{1} m_{3}(T-t)-2} .
\end{aligned}
$$

According to (a9), we have

$$
\begin{aligned}
D_{1}(t)= & \frac{k_{2}}{k_{1}} D_{2}(t)-\frac{k_{1} \lambda_{1}^{2}+2 k_{2}}{k_{1}}(T-t) \\
& +\frac{a k_{1}+b k_{2}}{k_{1}}\left(m_{3}(T-t)-\frac{2}{k_{1}} \ln \frac{2}{2-k_{1} m_{3}(T-t)}\right) .
\end{aligned}
$$

(iii) If $\Delta_{1}<0$, i.e.,

$$
\left(2 k_{1} \lambda_{2}-b\right)^{2}+2 k_{1}\left(2-k_{1} \lambda_{2}^{2}\right)<0 \Rightarrow \xi_{1}<\lambda_{2}<\xi_{2}, \quad b^{2}>4 k_{1},
$$

then (a4) has no real roots. But (a2) can be solved as follows.

$$
\begin{aligned}
& \dot{D}_{2}(t)=\frac{1}{2} k_{1}\left(\left(D_{2}(t)-m_{3}\right)^{2}+\frac{-\Delta_{1}}{k_{1}^{2}}\right) \\
& \Rightarrow \frac{\dot{D}_{2}(t)}{\frac{-\Delta_{1}}{k_{1}^{2}}\left(\left(\frac{k_{1}}{\sqrt{-\Delta_{1}}}\left(D_{2}(t)-m_{3}\right)\right)^{2}+1\right)}=\frac{1}{2} k_{1} \\
& \Rightarrow D_{2}(t)=m_{3}+\frac{\sqrt{-\Delta_{1}}}{k_{1}} \tan \left(\arctan \frac{-k_{1} m_{3}}{\sqrt{-\Delta_{1}}}-\frac{\sqrt{-\Delta_{1}}}{2}(T-t)\right) .
\end{aligned}
$$


According to (a9), we have

$$
\begin{aligned}
D_{1}(t)= & \frac{k_{2}}{k_{1}} D_{2}(t)-\frac{k_{1} \lambda_{1}^{2}+2 k_{2}}{k_{1}}(T-t) \\
& +\frac{a k_{1}+b k_{2}}{k_{1}}\left(m_{3}(T-t)-\frac{2}{k_{1}} \ln \cos \left(\arctan \frac{-k_{1} m_{3}}{\sqrt{-\Delta_{1}}}\right)\right. \\
& \left.+\frac{2}{k_{1}} \ln \cos \left(\arctan \frac{-k_{1} m_{3}}{\sqrt{-\Delta_{1}}}-\frac{\sqrt{-\Delta_{1}}}{2}(T-t)\right)\right) .
\end{aligned}
$$

The proof is completed.

(A2) The proof of Lemma 2 The following two cases are discussed.

(i) If $u-\lambda_{1} v=0$, it is obviously that (25) holds.

(ii) If $u-\lambda_{1} v \neq 0$, defining a variational operator for any function $g(t, r)$ as

$$
\nabla g(t, r)=-r g+\left(a-b r+\lambda_{2} \sigma_{r}^{2}\right) g_{r}+\frac{1}{2} \sigma_{r}^{2} g_{r r}
$$

we rewrite (24) as

$$
\frac{\partial g(t, r)}{\partial t}+\nabla g(t, r)+u-\lambda_{1} v=0, \quad g(T, r)=\eta .
$$

Noting that $g(t, r)=\left(u-\lambda_{1} v\right) \int_{t}^{T} \tilde{g}(s, r) \mathrm{d} s+\eta \tilde{g}(t, r)$, we derive

$$
\begin{aligned}
\frac{\partial g(t, r)}{\partial t} & =-\left(u-\lambda_{1} v\right) \tilde{g}(t, r)+\eta \frac{\partial \tilde{g}(t, r)}{\partial t} \\
& =\left(u-\lambda_{1} v\right)\left(\int_{t}^{T} \frac{\partial \tilde{g}(s, r)}{\partial s} \mathrm{~d} s-\tilde{g}(T, r)\right)+\eta \frac{\partial \tilde{g}(t, r)}{\partial t}, \\
\nabla g(t, r) & =\left(u-\lambda_{1} v\right) \int_{t}^{T} \nabla \tilde{g}(s, r) \mathrm{d} s+\eta \cdot \nabla \tilde{g}(t, r) .
\end{aligned}
$$

Putting (a19) and (a20) into (a18) yields

$$
\left(u-\lambda_{1} v\right)\left(\int_{t}^{T}\left(\frac{\partial \tilde{g}(s, r)}{\partial s}+\nabla \tilde{g}(s, r)\right) \mathrm{d} s-\tilde{g}(T, r)+1\right)+\eta\left(\frac{\partial \tilde{g}(t, r)}{\partial t}+\nabla \tilde{g}(t, r)\right)=0
$$

Thus, we get

$$
\frac{\partial \tilde{g}(t, r)}{\partial t}+\nabla \tilde{g}(t, r)=0, \quad \tilde{g}(T, r)=1 .
$$

In a result, we complete the proof.

(A3) The proof of Lemma 3 Inserting $\tilde{g}(t, r)=\mathrm{e}^{D_{3}(t)+D_{4}(t) r}$ into (25), we obtain

$$
\begin{aligned}
\mathrm{e}^{D_{3}(t)+D_{4}(t) r} & \left\{\dot{D}_{3}(t)+\left(\lambda_{2} k_{2}+a\right) D_{4}(t)+\frac{1}{2} k_{2} D_{4}^{2}(t)\right. \\
& \left.+r\left(\dot{D}_{4}(t)-1+\left(\lambda_{2} k_{1}-b\right) D_{4}(t)+\frac{1}{2} k_{1} D_{4}^{2}(t)\right)\right\}=0 .
\end{aligned}
$$

After separating the variables, we obtain the following two equations:

$$
\dot{D}_{4}(t)-1+\left(\lambda_{2} k_{1}-b\right) D_{4}(t)+\frac{1}{2} k_{1} D_{4}^{2}(t)=0, \quad D_{4}(T)=0 ;
$$




$$
\dot{D}_{3}(t)+\left(\lambda_{2} k_{2}+a\right) D_{4}(t)+\frac{1}{2} k_{2} D_{4}^{2}(t)=0, \quad D_{3}(T)=0 .
$$

Note that the discriminant $\Delta_{2}$ for the quadratic equation:

$$
-\frac{1}{2} k_{1} D_{4}^{2}(t)-\left(\lambda_{2} k_{1}-b\right) D_{4}(t)+1=0
$$

satisfies the condition $\Delta_{2}=\left(\lambda_{2} k_{1}-b\right)^{2}+2 k_{1}>0$.

Therefore, applying the same technique as the equation (a2), we derive

$$
D_{4}(t)=\frac{m_{4} m_{5}\left(1-\exp \left\{-\frac{1}{2} k_{1}\left(m_{3}-m_{4}\right)(T-t)\right\}\right)}{m_{4}-m_{5} \exp \left\{-\frac{1}{2} k_{1}\left(m_{3}-m_{4}\right)(T-t)\right\}},
$$

where $m_{4}$ and $m_{5}$ are two different roots. In addition, we have

$$
m_{4,5}=\frac{\lambda_{2} k_{1}-b}{-k_{1}} \pm \frac{\sqrt{\left(\lambda_{2} k_{1}-b\right)^{2}+2 k_{1}}}{-k_{1}} .
$$

Using the approach $(\mathrm{a} 23) \times k_{1}-(\mathrm{a} 24) \times k_{2}$, we obtain

$$
\dot{D}_{3}(t)=\frac{k_{2}}{k_{1}} \dot{D}_{4}(t)-\frac{a k_{1}+b k_{2}}{k_{1}} D_{4}(t)-\frac{k_{2}}{k_{1}} .
$$

Solving (a27), we get

$$
\begin{aligned}
D_{3}(t)= & \frac{k_{2}}{k_{1}} D_{4}(t)+\frac{k_{2}}{k_{1}}(T-t) \\
& +\frac{a k_{1}+b k_{2}}{k_{1}}\left(m_{5}(T-t)+\frac{2}{k_{1}} \ln \frac{m_{4}-m_{5}}{m_{4}-m_{5} \exp \left\{-\frac{1}{2} k_{1}\left(m_{4}-m_{5}\right)(T-t)\right\}}\right) .
\end{aligned}
$$

Therefore, the conclusion is proved.

(A4) The proof of Lemma 4 Considering the different expressions under different conditions, we will discuss the following cases:

(i) If $\left(2 k_{1} \lambda_{2}-b\right)^{2}+2 k_{1}\left(2-k_{1} \lambda_{2}^{2}\right)>0, D_{2}(t)$ is given by (a8). Differentiating $D_{2}(t)$ with respect to (w.r.t.) $t$, we have

$$
\dot{D}_{2}(t)=\frac{\left(k_{1} \lambda_{2}^{2}-2\right)\left(m_{1}-m_{2}\right)^{2} \exp \left\{\frac{1}{2} k_{1}\left(m_{1}-m_{2}\right)(T-t)\right\}}{\left(m_{1}-m_{2} \exp \left\{\frac{1}{2} k_{1}\left(m_{1}-m_{2}\right)(T-t)\right\}\right)^{2}} .
$$

Notice that under the condition of $k_{1} \lambda_{2}^{2}>2$, we have $\dot{D}_{2}(t)>0$. Therefore, $D_{2}(t)$ increases with respect to $t$, which leads to $D_{2}(t)<D_{2}(T)=0$.

(ii) If $\left(2 k_{1} \lambda_{2}-b\right)^{2}+2 k_{1}\left(2-k_{1} \lambda_{2}^{2}\right)=0, D_{2}(t)$ is given by (a13). Differentiating $D_{2}(t)$ w.r.t. $t$, we get

$$
\dot{D}_{2}(t)=\frac{2 k_{1} m_{3}^{2}}{\left(k_{1} m_{3}(T-t)-2\right)^{2}}>0 .
$$

Therefore, $D_{2}(t)$ is an increasing function w.r.t. $t$.

(iii) If $\left(2 k_{1} \lambda_{2}-b\right)^{2}+2 k_{1}\left(2-k_{1} \lambda_{2}^{2}\right)<0, D_{2}(t)$ is given by (a16). Differentiating $D_{2}(t)$ w.r.t. $t$, we have

$$
\dot{D}_{2}(t)=\frac{\left(\sqrt{-\Delta_{1}}\right)^{2}}{2 k_{1}} \sec ^{2}\left(\arctan \frac{-k_{1} m_{3}}{\sqrt{-\Delta_{1}}}-\frac{\sqrt{-\Delta_{1}}}{2}(T-t)\right)>0
$$


Therefore, $D_{2}(t)$ is also an increasing function w.r.t. $t$.

(iv) According to (a26), we have

$$
\dot{D}_{4}(t)=\frac{\left(m_{3}-m_{4}\right)^{2} \exp \left\{-\frac{1}{2} k_{1}\left(m_{3}-m_{4}\right)(T-t)\right\}}{\left(m_{3}-m_{4} \exp \left\{-\frac{1}{2} k_{1}\left(m_{3}-m_{4}\right)(T-t)\right\}\right)^{2}}>0 .
$$

Noting (a9) and (a27), we obtain

$$
\dot{D}_{1}(t)+2 \dot{D}_{3}(t)=\frac{k_{2}}{k_{1}}\left(\dot{D}_{2}(t)+2 \dot{D}_{4}(t)\right)-\frac{a k_{1}+b k_{2}}{k_{1}}\left(D_{2}(t)+2 D_{4}(t)\right)+\lambda_{1}^{2} k_{1} .
$$

Further, we find that $\dot{D}_{4}(t)>0$, so we have $D_{4}(t)<D_{4}(T)=0$.

Taking $\dot{D}_{2}(t)>0, \dot{D}_{4}(t)>0, D_{2}(t)<0$ and $D_{4}(t)<0$ into consideration, it is very obvious to get $\dot{D}_{1}(t)+2 \dot{D}_{3}(t)>0$, and we have $D_{1}(t)+2 D_{3}(t)<D_{1}(T)+2 D_{3}(T)=0$.

Therefore, Lemma 4 holds. 